\title{
A Chemical Theory of Topological Insulators
}

\author{
Angel Martín Pendás ${ }^{1}$, Julia Contreras-García ${ }^{2}$, Fernanda Pinilla ${ }^{3,4}$, José D. Mella ${ }^{3,4}$, Carlos \\ Cardenas $^{3,4}$, and F. Munoz 3 ,4 \\ ${ }^{1}$ Dpto. Química Física y Analítica. Universidad de Oviedo, Spain \\ ${ }^{2}$ Sorbonne Université and CNRS, 4 Pl Jussieu, 75005, Paris, Francia \\ ${ }^{3}$ Departamento de Física, Facultad de Ciencias, Universidad de Chile, Chile \\ ${ }^{4}$ CEDENNA, Santiago, Chile
}

January 23, 2019

\section{Abstract}

Research on topological insulators (TIs) has experienced an exponential growth in the last years, promising new technological applications in fields ranging from electronics to quantum computing. However, the strong condensed matter physical background that is needed to understand the exotic electronic structure of TIs has precluded its dissemination into the chemistry community. In this work we use chemistry-like models (e.g. the Hückel model) to bridge this gap. By taking bond alternating polyacetylenes as a starting point, we show how several key concepts about TIs, such as chiral symmetries or topologically-protected edge states, may be rephrased in terms of traditional chemical concepts by using Lewis resonance structures and bonding descriptors that characterize electron delocalization in real space. We will also show how covalent bond orders are able to discriminate between the trivial and topological phases of polyacetylenes.

\section{Introduction}

The last decade witnessed the field of condensed matter physics experience a revolution with the prediction $[4,5,18]$ and experimental realization $[9,34]$ of the socalled topological insulators (TIs) $[15,26]$. This new quantum state of matter instantly became a hot topic in other areas of physics and materials science $[1,14,30]$. In the chemistry community, however, topological insulators have not had the same widespread reception. While there have been several articles published in chemistry journals, their content has been mostly related to the physical or material science aspects of these systems. The aim of these articles has been to introduce TIs to chemists by presenting their phenomenology, exotic electronic structure, realizations in real-world materials and potential applications, but they have avoid delving into their theoretical characterization [7, 20,24]. Recently, Bradlyn et al. [6] have developed a theory of "topological quantum chemistry", which covers the full spectrum of topological materials, but which requires a strong physical background. Furthermore, this theory does not describe the chemistry behind these compounds.

One of the causes for the apparent lack of interest in topological materials on the part of chemists is the big gap between the language and the methods of reasoning used by condensed matter physicists and theoretical chemists. To begin with, the concept of topology in Condensed Matter Physics has nothing to do with the way topological analysis is interpreted in theoretical chemistry (i.e. the analysis of scalar fields such as the electron density in the framework of the quantum theory of atoms in molecules, QTAIM) [3]. In this article, we aim to scrutinize the simplest TI model, in order to provide thorough and detailed explanation of its theory from the point of view of a chemist, which would result in bridging the aforementioned gap.

TIs are insulators in the bulk, but with conducting states present on their surfaces. These surface states are pretty peculiar; they are topologically protected, in the sense that they remain conductive for as long as certain symmetries are preserved (we elaborate on the exact meaning of this statement in the next section). Moreover, the "protection" is independent of the surface cleanness, disorder, passivation, etc. Under all these conditions, the surface (or interface) states will remain conducting.

These unique features make TIs the subject of intense research for their application in low-power electronics [36], molecular-based spintronics [22], next-generation solar cells [37], quantum computing [12], photonics [19], novel organometalics [33], etc. Interestingly, the conducting surface states of TIs have attracted attention because of their ability to play the role of an electron reservoir that enhances the catalytic properties of noble metals supported on TIs $[8,28,35]$.

The most famous TIs belong to the $\mathrm{Bi}_{2} \mathrm{Se}_{3}$ family, which displays a single Dirac cone (i.e. electrons behaving like relativistic massless fermions) centred at $\Gamma$, the origin of the Brillouin zone. In this family of materials, the driving force of their topologically non-trivial fea- 
tures arises from a band inversion near the Fermi energy, which happens in turn due to the spin-orbit coupling (SOC) $[11,38]$. Without SOC, the valence and conduction bands of $\mathrm{Bi}_{2} \mathrm{Se}_{3}$ would be Se-derived and Bi-derived, respectively (in reciprocal space), and this would imply a normal insulator; however, the sizable $\mathrm{SOC}$ of $\mathrm{Bi}_{2} \mathrm{Se}_{3}$ exchanges the character of the valence and conduction bands near $\Gamma$. This band-inversion mechanism implies a non-trivial Berry phase. We call this particular kind of TIs $\mathcal{Z}_{2}$, and it can be shown that they need time-reversal symmetry in order to be preserved (e.g. no magnetic fields or impurities are allowed).

These are the kinds of concepts a chemist is exposed to when trying to understand TIs. The theory related to the $\mathcal{Z}_{2}$ subset of TIs is involved and it requires a heavy background in physics. However, simpler models more appealing to chemists do exist, which enable the understanding of the TIs' properties on chemical grounds. In this paper, we review the famous Su-Schrieffer-Heeger (SSH) model of polyacetylenes, providing some insight into their topological properties from standard chemical models and bonding descriptors.

\section{The Hückel Model of Poly- acetylene}

Polyacetylene is the simplest conjugated polymer, with chemical formula $(\mathrm{CH})_{2 N}$. It is formed by alternating blocks of -CH- groups coupled by single and double bonds. Su, Schrieffer and Heeger (SSH) provided a very simple characterization of polyacetylenes by means of a tight-binding model [29]. An excellent introduction to the topological features of this model is given by Ásboth et al. [2] Here, we adapt it to a Hückel formulation as well as to wide-spread chemical concepts.

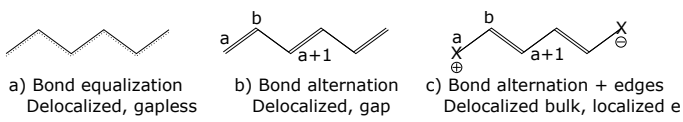

Figure 1: Chemical representation of the resonance forms of interest in 1,3,5-hexatriene

Within the Hückel formalism, conjugated systems are usually treated by ignoring hydrogen atoms and considering only a single orbital, $\phi_{i}$,-and a single electron- per carbon atom. Conjugation is introduced by means of a set of alternating single and double bonds, and only nearest-neighbour interactions are considered. Under these approximations, the effective one-electron Hamiltonian matrix elements are $\alpha$, the on-site energy and $\beta$, the nearest-neighbour interaction:

$$
\begin{gathered}
\alpha=-\left\langle\phi_{i}|H| \phi_{i}\right\rangle \\
t=-\left\langle\phi_{i}|H| \phi_{i+1}\right\rangle
\end{gathered}
$$

This minimal model provides a rather good description of aromatic molecules (e.g. benzene), where the reso- nance between different Lewis structures leads to bond equalization (see Fig. 1a). This is not the case in conjugated linear molecules, such as polyacetylene, where bond alternation is observed. To overcome this deficiency, it is possible to introduce two different nearestneighbour interactions $\beta$ and $\beta^{\prime}$ (see Fig. 1b) :

$$
t=-\left\langle\phi_{i, a}|H| \phi_{i, b}\right\rangle, t^{\prime}=-\left\langle\phi_{i, b}|H| \phi_{i+1, a}\right\rangle,
$$

The subscripts $a, b$ refer to the new symmetry of the system. Indeed, upon bond alternation, the unit cell of the polymer is doubled. Under this scenario, we can observe two inequivalent carbon positions: those having the double bond to their right, and those having it to their left. Hence, in the $i$-th unit cell there are two carbon atoms, labelled $a_{i}$ and $b_{i}$, that contribute to the bond-alternated Hückel Hamiltonian with $\phi_{i, a}, \phi_{i, b}$ orbitals (see Fig. 1b). Assuming the same notation as in Fig. $1 \mathrm{~b}$, we intend $\beta$, the interaction between $a_{i}$ and $b_{i}$, to represent the double bond interaction, whereas $\beta^{\prime}$ in between $b_{i}$ and $a_{i+1}$ is intended to represent the single bond (i.e. $\beta>\beta^{\prime}$ ).

In what follows, we will ignore the on-site energy $(\alpha=$ 0 ), which is valid as long as it has the same value for each atom (i.e. all atoms are equivalent). With this in mind, the Hückel-like Hamiltonian of polyacetylene can be written as

$$
H=-\left(\begin{array}{cccc}
0 & \beta & 0 & 0 \cdots \\
\beta & 0 & \beta^{\prime} & 0 \cdots \\
0 & \beta^{\prime} & 0 & \beta \cdots \\
0 & 0 & \beta & 0 \cdots \\
\vdots & \vdots & \vdots & \ddots
\end{array}\right)
$$

A direct diagonalization of $H$ without using periodic boundary conditions gives two qualitatively different outcomes.

The first one is known within the physics community as the normal or trivial case, $\beta>\beta^{\prime}$; it corresponds to the resonance form with the biggest weight since it has no charges (Fig. 1b). In this regime, there is a clear energy gap between the highest occupied (HOMO) and the lowest unoccupied (LUMO) molecular orbitals (Fig. 2a left). In the case of infinite systems this bandgap is given by $2\left(\beta-\beta^{\prime}\right)$. Orbitals display the usual delocalized behavior we would expect for a conjugated $\pi$ system (Fig. 2a right).

As $\beta \rightarrow \beta^{\prime}$ and the distinction between single and double bonds vanishes (i.e., $\beta=\beta^{\prime}$ ), the model falls back into the common Hückel approach with bond equalization (Fig. 1a). For very large chains, this means that the system becomes (virtually) gapless (metallic) (see S.I. for the band gap and frontier molecular orbitals).

Now we can assume, just like with benzene, that conjugated double bonds can easily lead to resonance and study the structure represented in Fig. 1c. First of all, this leads to a new notation for the interactions, since we now have a single bond between $a_{i}$ and $b_{i}$, and a double bond between $b_{i}$ and $a_{i+1}$. Keeping the same notation as 


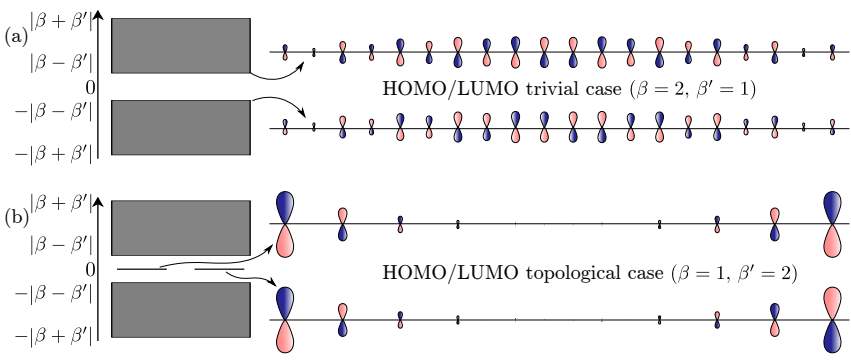

Figure 2: Energy bands (left) and HOMO/LUMO coefficients (right) for the SSH solutions of $(\mathrm{CH})_{20}$. (a) trivial solution $\left(\beta>\beta^{\prime}\right)(\mathrm{b})$ topological solution $\left(\beta<\beta^{\prime}\right)$

in Eq. 3, this is equivalent to saying that $\beta^{\prime}>\beta$. Mathematically, this constitutes a second family of solutions.

But why is this a qualitatively different family? The resonance form where $\beta^{\prime}>\beta$ has two edges (Fig. 1c). Recall that only $\pi$ bonds are considered within the Hückel approach. Two new energy levels appear on top of the previous energy distribution, located in the middle of the band gap (that is at zero-energy, see Fig. 2b-left); these become the new HOMO and LUMO orbitals of the system, which are non-bonding. Since they do not mix with the rest of the chain, they remain very localized. Fig. 2b-right shows the corresponding orbitals. It can be seen that, unlike the orbitals in the rest of the chain, the two new ones do not delocalize over the bulk, but remain rather localized on the edges. Moreover, since they are formed from just two edges, a bonding and anti-bonding pair is formed (the phase opposition can be seen toward the right border). Note that the overlap between the atomic orbitals (located on opposite edges) that contribute to these two molecular orbitals is very small, which explains why they virtually have the same energy. These states are rather special: their existence is independent of the actual value of $\beta$ and $\beta^{\prime}$ (as long as $\beta^{\prime}>\beta$ ). This fact explains the "protection" of topological insulator properties upon changes in $\beta$ and $\beta^{\prime}$, i.e. upon chemical changes. The second solution of the SSH model can be understood as the simplest case of topologically protected edge states.

In the remainder of the paper we elaborate on the existence and properties of such states, which can be experimentally realized in the solid state and whose properties can in many cases be explained and deduced from this simple model.

\subsection{The bipartite lattice and the chiral symmetry}

Why does this model of polyacetylenes show protected zero-energy edge states? The answer resides in its bipartite lattice, i.e. in bond alternation. From the point of view of Solid State, there are two equivalent sites per unit cell, but which are not connected by a lattice vector (but by a non-Bravais vector). Then, what makes the bipartite lattice so special? It is the fact that it possesses an extra symmetry, known as chiral symmetry. We further explain what this means from a chemical viewpoint.

\subsubsection{The lattice is formed by two sub-lattices}

Let us return to the Hamiltonian matrix $H$ (Eq. 4). Since the labelling of the atomic orbitals is irrelevant (i.e. the Hamiltonian operator is symmetric under the permutation of the electrons), a rearrangement of the atomic labelling such that all atomic orbitals belonging to sublattice $a$ come before those of sub-lattice $b$ puts the $H$ matrix into an explicit block-diagonal form:

$$
H=\left(\begin{array}{cc}
0 & H_{a b} \\
H_{a b}^{T} & 0
\end{array}\right),
$$

where the wave-vector has been rearranged in $a$ and $b$ blocks: $\Psi=\left(\phi_{0, a}, \phi_{1, a}, \phi_{2, a} \ldots \phi_{N-1, b}, \phi_{N, b}\right)$. The possibility of blocking the Hamiltonian stems from the absence of on-site energies in the same sub-lattice, i.e.: $\alpha_{a}=\alpha_{b}=\left\langle\phi_{i, a}|H| \phi_{i, a}\right\rangle=\left\langle\phi_{i, b}|H| \phi_{i, b}\right\rangle=0$, and leads to the sublattices $a$ and $b$ being manifestly split. The projection operators onto each sub-lattice, $P_{a}$ and $P_{b}$, satisfy $P_{a}+P_{b}=1$ and $P_{a} P_{b}=0$. The chemical meaning of these relationships is revealed further below.

$P_{a}+P_{b}=1$ means that the whole system is formed by centres which are either "a" or "b". $P_{a} P_{b}=0$ is extremely related to resonance. If a charge is situated on a given center $b_{i}$, resonance forms will only delocalize the charges along the $b_{i}(i=1, N)$ centres (Fig. 3a). Of course the same applies to the $a$ sub-lattice. This result is well known within aromatic compounds in chemistry (Fig. 3b). A charge located on a benzene ring in the ortho position ( $b_{1}$ in Fig. $3 \mathrm{~b}$ ) will be delocalized over other para (position $b_{2}$ ) and ortho $\left(b_{3}\right)$ positions, but not over the meta $\left(a_{2}, a_{3}\right)$ positions.

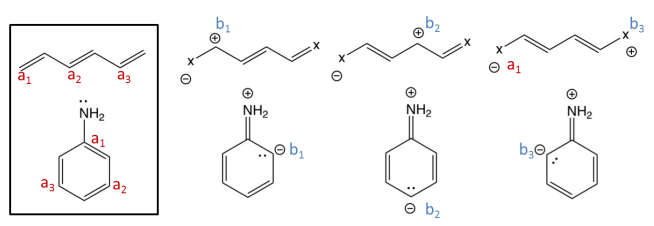

Figure 3: a)Delocalization of charges in the bulk of one sub-lattice for hexatriene b) Delocalization of charges in aniline

More generally, the above can be easily verified if we look at the delocalization indeces, $\delta_{i j}$, which provide a measure of the delocalized population between centres $i$ and $j$. In naïve cases (such as Hückel) $\delta_{i j}$ may be simply taken as the bond order (a more detailed explanation on delocalization indeces is given in S.I). In our bipartite lattice, $i$ and $j$ can belong indistinctively to either the $a$ or the $b$ sub-sets. For example, a delocalized cyclic form (to avoid borders) like that of Fig. 1a in cyclohexatriene (i.e. benzene) leads to nearest-neighbour $\delta_{i, i+1} \simeq 1.4$ for $i \in 1, N-1$, whereas a localized one, like in Fig. 1b, leads to $\delta_{a_{i} b_{i}} \simeq 2$ and $\delta_{a_{i+1} b_{i}} \simeq 1$. 
For our purpose, it is important to note that DIs decay exponentially for insulators and in a power-law manner for metals, [13] and this relation is analytical in the case of tight-binding models. In other words, DIs reflect the fact that in the metallic case electrons are more delocalized. DIs have also been found to be related to Resta's [23] localization tensor, meaning that their decay rate is rigorously related to the modern theory of polarization.

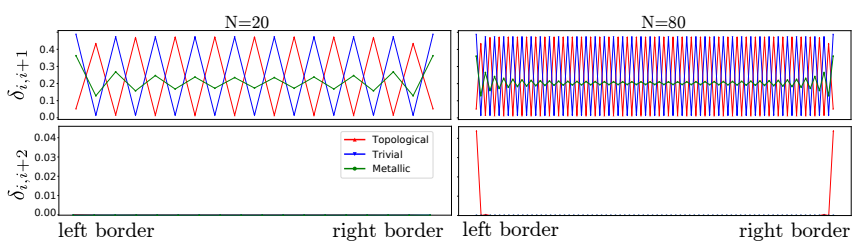

Figure 4: Delocalization Indices $\delta_{i, i+1}, \delta_{i, i+2}$ of the bipartite lattice. The chains are 20 (left) and 80 (right) atoms long. Three cases with different topology are shown: the trivial phase (in red) has $\beta=3$ and $\beta^{\prime}=1$, the topological phase (in blue) has those values reversed and the metallic phase (in green) has $\beta=\beta^{\prime}=2$

The delocalization index for two different types of bipartite chains, short (20 atoms) and long (80 atoms) of the SSH model is shown in Fig. 4. In both cases, the topological and trivial solutions were considered. In order to verify that chirality leads to charges that are only being delocalized on the complementary sub-lattice, second-neighbour delocalization needs to be analysed (i.e. $\left.\delta_{i, i+2}\right)$. As seen in the simple model in Fig. 3 , in the bulk-like region of the bipartite lattice $\delta_{1,3}$ is zero. Moreover, this happens regardless of the phase: trivial, topological or metallic. At the borders of the molecule, the behaviour is more interesting: $\delta_{1,3}=0$ for small chains regardless of the topological phase - a consequence of a small but non-negligible interaction between the borders. For longer chains, the border states are effectively decoupled and $\delta_{1,3} \neq 0$ just for border states.

It should be noted that it is usually very tricky to state whether two border states are decoupled or not. Rigorously, this can be achieved by having just one border (semi-infinite boundary conditions), which is hard to implement. This question is often overlooked and one relies on intuition or common sense. But we have found that chemical approaches such as bond orders from DIs $\left(\delta_{i, i+2}\right)$ are able to provide a quantitative answer: for the hopping strengths shown in Fig. 4, the crossover from short to long chains happens at $N \sim 70$ atoms, which is roughly $10 \mathrm{~nm}$. [21] Experiments with 3D topological insulators show that crossovers from coupled to decoupled surface states occur at a slab thickness of ca. $6 \mathrm{~nm}$. [40]

The two phases (trivial and topological) lead to different bulk-like features in the first neighbour DI, $\delta_{1,2}$ (the rightmost side of Fig. 4). The position of the local maxima and minima of the nearest-neighbour $\delta_{1,2}$ for the two solutions are opposed. This corresponds to the fact that the delocalized $\pi$ electrons have changed posi- tion. Whereas the $\pi$ bond is located in between $a_{i}$ and $b_{i}$ in the trivial phase, in the topological phase they are located in the complementary set $\left(b_{i}\right.$ and $\left.a_{i+1}\right)$ (recall that $\left.P_{a}+P_{b}=1\right)$. With respect to $\delta_{i, i+1}$ at the molecule borders, the trivial edge states are more localized than those of the non-trivial case. In both situations the localization of the edge quickly decays to its asymptotic bulk value.

Hence, the simultaneous analysis of delocalization patterns, $\delta_{i, i+1}$ and $\delta_{i, i+2}$, enables us to identify a chiral setup along with the different phases. While the former informs us on the existence of resonance, the latter confirms the existence of a phase with two edges, i.e. of a topological phase and chiral symmetry.

It is also interesting to analyze $\delta_{i, i+1}$ in the intermediate metallic case $\left(\beta=\beta^{\prime}\right)$. Here, it is easy to see how the metallic behaviour differs from the localized case. Whereas the decay in the non-metallic states $\left(\beta \neq \beta^{\prime}\right)$ $\delta_{i, i+1}$ decays exponentially, the metallic state shows the typical polynomial decay. [13] This approach makes it possible to identify from the wavefunction the localization schemes expressed in Fig. 1. While the $\pi$ electrons are delocalized for $\beta=\beta^{\prime}$ (Fig. 1a), they are localized for $\beta \neq \beta^{\prime}$ (Figs. 1b,c). From the computational point of view, localization schemes (e.g. Foster-Boys) would lead to a unique answer in the $\beta \neq \beta^{\prime}$ case, similar to the bulk molecular orbitals in Fig. 2c. Instead, the localization for $\beta=\beta^{\prime}$ would not be unique.

It is clear that transforming the trivial phase $\left(\beta^{\prime}>\right.$ $\beta$ ) into the topological one $\left(\beta^{\prime}<\beta\right)$ or viceversa in a continuous way (i.e. adiabatically) implies closing the gap $\left(\beta^{\prime}=\beta\right)$. This implies crossing through a situation with fully equalized bonds, which in the limit $\beta \rightarrow 0$, means passing through an atomic situation (see S.I. for a more in-depth explanation).

\subsubsection{Energies come in pairs}

Chirality also implies important consequences on the MO diagram of the system. If $\psi$ is a solution of the oneelectron Schrödinger equation, $H \psi=E \psi$, there must be another solution with opposite eigenvalue, $-E$ (see S.I. for more details). Therefore all states must come in pairs with energy values $\{E,-E\}$. These are called chiral partners.

This property is also preserved in the $\beta=\beta^{\prime}$ limiting case. Let us briefly examine a very well known case in chemistry, the Hückel solution of butadiene (the simplest $2 \mathrm{~N}$ conjugate hydrocarbon) at $\alpha=0$. The energies and molecular orbitals of the four one-electron states are given by:

$$
\begin{array}{ll}
E_{1}=+1.6 \beta, & \pi_{1}=0.37 \phi_{1}+0.6 \phi_{2}+0.6 \phi_{3}+0.37 \phi_{4} \\
E_{2}=+0.6 \beta, & \pi_{2}=0.6 \phi_{1}+0.37 \phi_{2}-0.37 \phi_{3}-0.6 \phi_{4} \\
E_{3}=-0.6 \beta, & \pi_{3}=0.6 \phi_{1}-0.37 \phi_{2}-0.37 \phi_{3}+0.6 \phi_{4} \\
E_{4}=-1.6 \beta, & \pi_{4}=0.37 \phi_{1}-0.6 \phi_{2}+0.6 \phi_{3}-0.37 \phi_{4}
\end{array}
$$

It can be observed how $E_{1}=-E_{4}$ and $E_{2}=-E_{3}$, correspond to the two chiral pairs. They are related by 
a change of sign on the $b$ sublattice $\left(\phi_{2}, \phi_{4}\right)$, which transforms the chiral pairs: $\pi_{1}$ into $\pi_{4}$, and $\pi_{2}$ into $\pi_{3}$. This general rule for constructing energies and molecular orbitals within the Hückel approach is a direct consequence of the chiral symmetry of the even number of carbons chain.

As we saw before, these MOs are delocalized over the whole system and have support (non-zero coefficients) on both sub-lattices. This is apparent in the butadiene example as well as in bigger polyacetylenes. Fig. 2a shows how the MOs are delocalized over the whole unit, with both the HOMO and the LUMO displaying contributions in both sub-lattices. However, this is not the case at the borders. When a state has zero-energy, $E=-E=0$, the chiral partners become degenerate and they only have support on one of the sub-lattices, either $a$ or $b$. This is easily seen in the leftmost structure of Fig. 3a. Due to the pairing, one edge belongs to the $a$ sub-lattice and the other edge belongs to the $b$ sub-lattice. Hence, the state on the left (right) has non-vanishing contributions only in the $a_{i}\left(b_{i}\right)$ sites.

Graphene is a system similar to polyacetylene in 2D. Its hexagonal lattice is bipartite (two $\mathrm{C}$ atoms in each cell), making it a "chiral" material. Unlike polyacetylene, all the bonds in graphene have the same strength $\left(\beta=\beta^{\prime}\right)$, making it a very special conductor.

\subsection{So where does "chirality" come into play?}

In order to obtain the peculiar properties of a topological phase, chirality needs to be set up: an even number of centres with zero on-site energy. When one of these requirements is switched off, chirality is lost. We illustrate the consequences of this setyp with two simple examples.

\subsubsection{The bipartite lattice}

If we take a system with an odd-number of conjugated carbons, the $(E,-E)$ correspondence breaks down. For example, the Hückel model for the allyl cation (3 centres) under bond equalization conditions and $\alpha=0$, would lead to

$$
\begin{aligned}
E_{1}=-\sqrt{2} \beta, & \pi_{1}=\phi_{1}+\sqrt{2} \phi_{2}+\phi_{3} \\
E_{2}=0, & \pi_{2}=-\phi_{1}+\phi_{3} \\
E_{3}=\sqrt{2} \beta, & \pi_{3}=\phi_{1}-\sqrt{2} \phi_{2}+\phi_{3}
\end{aligned}
$$

Molecular orbitals $\pi_{1}$ and $\pi_{3}$ are connected by a change of sign in the $a$ and $b$ sub-lattices. One nonbonding orbital does appear $\left(\pi_{2}\right)$, but it is not protected, since it has no partner imposed by symmetry.

The normal Hückel solution for allyl cation involves $\beta=\beta^{\prime}$. However, the consequences of the non-bipartite lattice go even further when we analyse $\beta \neq \beta^{\prime}$. Fig. 5 shows the results for the energy and the frontier molecular orbitals for $N=21\left(\beta \neq \beta^{\prime}\right)$. Since we have an odd number of $\pi$ electrons, the highest occupied molecular orbtital is singly occupied (SOMO for Singly Occupied

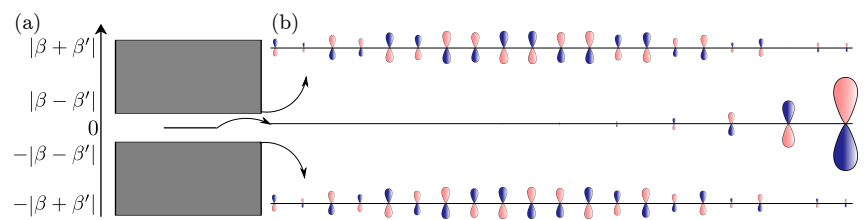

Figure 5: Chain with 21 atoms, $\beta=2$ and $\beta^{\prime}=1$. (a) Energy diagram. (b) Orbitals close to the Fermi level. Reversing $\beta, \beta^{\prime}$ just localizes the zero-energy level to the left side.

Molecular Orbital). The non-bonding state is localized just on one end.

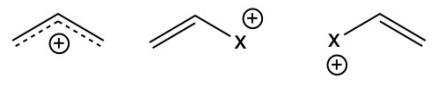

Figure 6: Resonance scheme for allyl cation

This can be understood if we analyse the resonance forms of the allyl cation. Since the number of carbon atoms is even, only one border appears. The resonance form in Fig. 6-center is the solution for $\beta>\beta^{\prime}$ whereas the resonance form in Fig. 6-right corresponds to $\beta^{\prime}>\beta$. It can be seen that, contrary to what we had found for a bipartite network, these two solutions are equivalent, so that we do not have two sets of solutions. Hence, the absence of a bipartite lattice destroys the existence of two different solutions or "phases". Indeed, the delocalization index $\delta_{1,3}$ (see Fig. 7) is non-zero only at one border -the position of the non-bonding atom. Increasing $\beta$ to a value larger than $\beta^{\prime}$ just changes the position of the non-bonding atom to the other border. The index $\delta_{i, i+1}$ at one border of the molecule behaves in the same way as in the trivial phase (Fig. 4), and like the topological phase at the other border, confirming the absence of two differentiated phases.

As a side note, the $\beta=\beta^{\prime}$ solution in Eq. 12 (Fig. 6a) corresponds to a delocalized SOMO along with a complete delocalization of the charge. For a large number of atoms this would lead to a metallic system just like that of the bipartite case. .

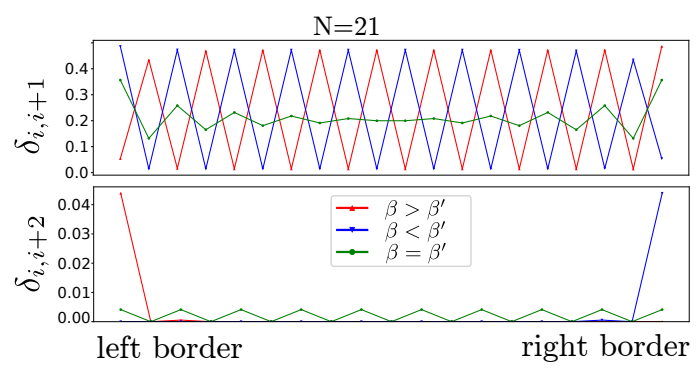

Figure 7: (a) $\delta_{i, i+1}$ (b) $\delta_{i, i+2}$, for a chain with $N=21$ atoms 


\subsubsection{The on-site energy}

The chirality is also broken upon distortion of the same on-site energy. This can be easily verified by changing the on-site energy of the atoms in one of the sub-lattices (i.e. by adding a difference of potential $\Delta V$ between atoms in positions $a_{i}$ and $b_{i}$ ). This term breaks the bipartite and chiral symmetries so that the Hamiltonian is no longer given by Eq. 5, but by the following matrix:

$$
H=\left(\begin{array}{cc}
0 & H_{a b} \\
H_{a b}^{T} & \Delta V
\end{array}\right)
$$

This is equivalent to considering a network of different atoms, type $A$ atoms on sites $a_{i}$ and type $B$ ones on sites $b_{i}$; for example, a (-C-N-) ${ }_{n}$ chain instead of polyacetylene (Fig. 8b). The potential, $\Delta V$, means that different atoms have different on-site energies, i.e. they have different values of electronegativity. This leads to resonance possibilities with charges held both on the $a$ and the $b$ sub-lattices (Figs. 8a,c). Fig. 8b corresponds to the resonance found in polyacetylene, which yields $\delta_{i, i+2}=0$. The different probability of holding charges on the different atoms, $\mathrm{C}$ and $\mathrm{N}$, gives rise to additional possibilities of delocalization so that charges delocalize over the full chain, both on $a$ and $b$ centres (Fig. 8a and c). a)

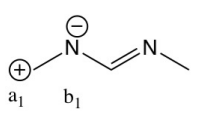

b)

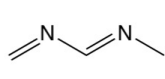

c)

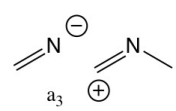

Figure 8: Delocalization of charges when $\alpha_{a} \neq \alpha_{b}$ (i.e. $\Delta V \neq 0)$.

This can be easily verified by returning to delocalization indeces. Fig. 9 shows $\delta_{1,2}$ and $\delta_{1,3}$ for $\alpha_{a} \neq \alpha_{b}$. As we have seen, resonance effects are reflected on $\delta_{i, i+2}$. Instead of the zero value obtained when chiral symmetry was present, in this case $\delta_{i, i+2}$ is finite everywhere in the bulk (Fig. 9 bottom). Instead, the $\delta_{i, i+1}$ index (Fig. 9 top) is qualitatively unaffected by the breaking of the chiral symmetry. In other words, we show that secondneighbour bond orders enable the effective identification (and quantification) of the absence of chiral symmetry.

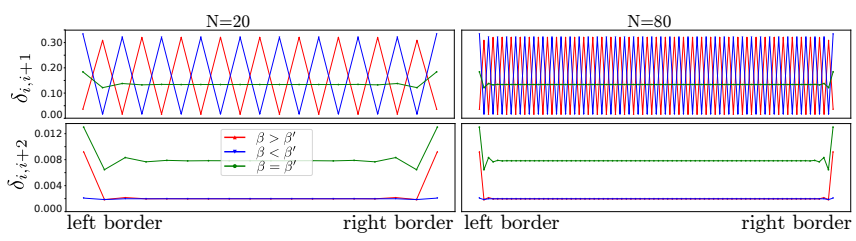

Figure 9: Delocalization Indices, $\delta_{i, i+1}$ and $\delta_{i, i+2}$, of the alternating di-atomic lattice, an on-site term $(\Delta V=-2)$ was added to the second sublattice. The chain is 20 and 80 atoms long and the hoppings $\beta, \beta^{\prime}$ are the same as in Fig. 4

\subsection{Topological phase transitions}

Why is the $\beta^{\prime}>\beta$ set of solutions called topological? Topology is the branch of mathematics concerned with the study of space and its continuous transformations. For instance, an orange is a body with no holes. We can easily imagine a continuous transformation from an orange shape to a dish shape (just by smashing a sphere of Play-Doh $\left.{ }^{(}\right)$. This means they are topologically equivalent, an equivalency that is identified by the fact that none of them has holes. A donut has as many holes as a coffee cup. Hence, they are topologically equivalent. Putting this together, the donut and the orange belong to different topological classes. This equivalency is represented by a number, the number of holes or genus. The fact that a different number of holes is allowed, enables the existence of "topological phases".

The existence of chirality in the $\mathrm{SSH}$ model ensures the possibility of a topology onset on the electronic structure, i.e. the possibility of having different phases, each of them characterized by a genus, and within which changes can occur in a continuous manner. Looking at the hexatriene in Fig. 1a, we could progressively stretch it (i.e. making $\beta^{\prime}$ progressively smaller) to make 3 double bonded dimers. It would also be possible to progressively stretch the dimers, leading to monomers at the $\beta \rightarrow 0$ limit. In a physicists' jargon, this would be equivalent to vacuum. All these $\beta>\beta^{\prime}$ possibilities correspond to the same $\beta>\beta^{\prime}$ set of solutions. They constitute one topological phase, in analogy to the equivalence between a dish and an orange.

The other topological phase of the model has $\beta<\beta^{\prime}$. Taking the resonance form from Fig. 1c, if we now stretch the single bonds (decrease $\beta$ ), we end up with two double bonded dimers and two borders, which corresponds to a different topological phase, like the donut in the analogy. The link between both phases occurs for all the $\beta=\beta^{\prime}$ cases.

What plays the role of genus in the electronic structure? A winding number, $\gamma$, can be defined in this case (see S.I. for more details), which will be 0 for the trivial case $\left(\beta^{\prime}>\beta\right)$ and 1 for the topological case $\left(\beta^{\prime}<\beta\right)$. The marginal case $\beta=\beta^{\prime}$ does not have a well-defined winding number.

What are the consequences of each topological phase? The fact that two phases are possible (unlike in the allyl cation case), leads to regions of different $\gamma\left(\beta>\beta^{\prime}\right.$, $\left.\beta<\beta^{\prime}\right)$ which are hence symmetry protected. There are just two ways of undefining $\gamma$. The first option is to have zero-energy states, which means changing from one topological phase to another crossing through $\beta=\beta^{\prime}$ and hence passing through a metallic state. The other option is to break the chiral symmetry. Regarding our Hamiltonian, this would mean including a term on its diagonal like it was done we did in Section 2.2.2, when different atoms were introduced.

It should be noted that the edge states must not necessarily be located on the extremes of the chain. For instance, it is easy to write resonance forms involving 
bulk carbon atoms, which introduce the so-called domain walls. In Fig. 10b, the polyacetylene chain can be (conceptually) split into two sub-systems: on the one side of the domain wall the system has a trivial winding number $(\gamma=0)$, while this number is not trivial $(\gamma=1)$ on the other side. Following the same reasoning used in our discussion of edge states, this resonance structure unavoidably leads back to non-bonding (zeroenergy) states, which hold the same properties as introduced before. They are localized at the interface or domain wall (see S.I. for more details) and become nonzero only in one sub-lattice. These zero-energy states are also topologically protected. The values of $\beta$ and $\beta^{\prime}$ can change, even the position of the limit in between the wall, but both domains will still coexist unless a phase transition occurs by changing $\gamma$.

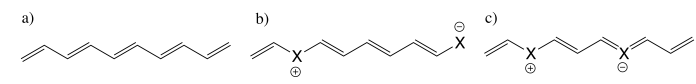

Figure 10: Resonance structures leading to domain walls: (a) regular bipartite lattice, (b) lattice with one domain wall and one non-bonding atom and (c) lattice with two domain walls. Each domain wall separates a region with "topological" order from one with "trivial" order.

\section{From the SSH model to real systems}

The Su-Schrieffer-Heeger (or SSH) model was proposed to explain the unusually high conductivity of conjugated polymers. Several of the concepts uncovered here are briefly summarized in the Nobel Lecture given by Heeger (Chemistry Nobel prize 2000 [16]), but without relating them to the topological phases of matter or their chemistry. The concept of "topological insulator" was coined much later, in 2007.

We now convey how the SSH model can assist in understanding the properties of real topological insulators. TIs are insulator materials in the bulk, but with conducting states at their surfaces. However, these surface states have very peculiar properties: they are topologically protected, in the sense that they remain conducting as long as the protecting symmetry is preserved (for example, the chiral symmetry we have seen). The surface (or interface) states remain conductive independently of the surface cleanness, disorder, passivation, etc. Moreover, scattering and dissipation are heavily suppressed in these states.

\subsection{Two-dimensional SSH-model}

Extending the concepts developed here with the SSH model to two- or three-dimensions is straightforward (but lengthy). However, we can use hand-waiving arguments to understand the general behaviour of TIs. Let us assume we make an equidistant array of SSH hydrocarbons and place them at a medium interaction distance. If each SSH-like strand is in the trivial phase, no edge state is to be expected, and the result is a trivial $2 \mathrm{D}$ insulator. Instead, if the chains are in the topological phase, the $2 \mathrm{D}$ object will have two regions:

- The bulk of the hydrocarbon chains. Since chains are far apart, neighbouring chains will only lead to weak interactions that slightly perturb the local electronic structure. This can be viewed just like a stacking of polyacetylene molecules, where the $\pi$ stacking dominates, but the general molecular orbital scheme is unaltered. Hence, they remain insulating. This part will be just the same as the bulk in the SSH model (localized, with bond alternation, localizable orbitals and polynomial $\delta_{i, i+1}$ decay)

- The borders. These are isolated "atoms", all placed at the same distance. If $\beta_{2}$ represents the interaction related to this new dimension, it results that all the border atoms interact with the same $\beta_{2}$ value. This indicates that a delocalized interaction (metallic state) is settled in between the edges.

Hence, we retrieve TIs protected characteristics: insulating bulk and conducting surface, which must remain metallic - unless the chiral symmetry is broken. Why are the topologically protected edges states so robust? The interaction parameters $\left(\beta, \beta^{\prime}\right)$ can be changed at will, but the winding number in the chains has to remain $\gamma=1$ if the phase is left unchanged. In this manner, we can introduce disorder, next-next-neighbour interactions, etc. and the edge states have to remain present.

Although these arguments are hand-waiving, they are meant to give an idea of the general features of topologically protected states, which can be transposed to real-world examples such as graphene [10] and blackphosporus nanoribbons. [31]

Unfortunately, the above holds only as long as the chiral symmetry is not broken. But we have seen that an atom with different electronegativity leads to the breaking of the chiral symmetry. Therefore, chemisorption of foreign atoms in these models ends with the TI state, which is not the case for the most commonly used TIs. This happens because these systems set up their topology with a symmetry that is resilient to doping.

\subsection{Topological insulators with time- reversal symmetry}

To find a more robust symmetry, we restate the sublattice symmetry of a conjugated polymer. Instead of using the chiral symmetry, given by "there are two identical but inequivalent atoms per unit cell", we make the following slight change to the statement: "there are two identical but inequivalent spins per unit cell". After replacing the sub-lattices $a, b$ with the spins up, down the analogy in the Hückel formulation is straight forward. To 
have the chiral symmetry in the SSH model both sublattices must be identical (i.e. the same electronegativity). The new spin-based symmetry is rather translated into: the total bulk spin must be zero (spin unpolarized) or, as physicists call it, the time-reversal symmetry has to be preserved. The use of spin -instead of the sublattice degree of freedom- is very convenient, since (i) the spin Hamiltonian will be just a $2 \times 2$ matrix (see Eq. 5), regardless of the size and dimensions of the unit cell, (ii) to break the time-reversal symmetry, a magnetic field or magnetic impurity is needed, otherwise the topological features will hold. In other words, non-magnetic impurities will not destroy the metallic surface states. $[25,32]$

In some materials with heavy elements, such as $\mathrm{Bi}_{2} \mathrm{Se}_{3}$, $\mathrm{Bi}_{2} \mathrm{Te}_{3}$ and $\mathrm{Sb}_{2} \mathrm{Te}_{3}$, the spin-orbit coupling is large enough to change the nature of the wavefunction, mixing the valence and conduction band. This triggers the transition to a topological state. The surface states ("edges") of these materials are spin polarized in reciprocal space; just like the SSH model topological phase had a net dipole (charges + and - ) in the edges/interfaces. Hence, instead of having two split sub-lattices, now we have a spin-split system. Still, the net spin moment averages to zero (just like the charges did in the SSH model), preserving the time-reversal symmetry. This means that it is possible to set up the system such that different current senses have different spins, so that the "turning around" must also flip the spin. The result is that dissipation in these states ends up being heavily suppressed, making the proposed time-reversal TIs a promising venture for spin-based electronics [17].

More specifically, these systems are especially useful in low-energy consumption electronics [36], molecularbased spintronics [22], next-generation solar cells [37], quantum computing [12], photonics [19], novel organometallics [33], and many more. Most recently, the conducting surface states of TIs have also attracted attention because they could play the role of an electron reservoir that enhances the catalytic properties of TIsupported noble metals $[8,27,28,35,39]$.

\section{Conclusions}

While the prediction and experimental realization of topological insulators constituted a revolution in material science, the language gap between physicists and chemists has precluded the expansion of the field in the chemical literature. We hereby show that the main concepts involving topological insulators can be understood by introducing a slight variation of the familiar Hückel formulation.

Starting from one of the simplest TI models, the SuSchrieffer-Heeger description of polyacetylene, and solving it with a Hückel-type of approach where bond alternation is possible, we have built a bridge between the physical and chemical description of TIs. Within this approach, two solutions appear. The first one is the normal conjugated system, which corresponds to the so- called "trivial phase". The other solution is a simple model of a "topological phase": it corresponds to the resonance form where charges are created on terminal atoms. These two extreme forms are connected by a delocalized (metallic) system with bond equalization (i.e., the typical Hückel Hamiltonian).

The existence of these two phases is due to an extra symmetry in the polyacetylene system in the case where bond alternation is allowed, known as "chiral symmetry". It sums down to two characteristics: the network is entirely made of the same atoms, and all the $\pi$ electrons come from double bonds, so that the chain has $2 \mathrm{~N} \pi$ electrons. The above two features ensure that everything comes in pairs: all atoms have a "partner" (through the double bonds), and so do eigenvalues (energies) and eigenvectors (molecular orbitals). This simple model enables us to understand the meaning of edge states, topological protection and other basic concepts in the theory on TIs -all in terms of standard chemical concepts like the Lewis resonance structures and electron delocalization. We also show that basic bonding descriptors, such as covalent bond orders (delocalization indeces), can be used to discriminate topologically non-trivial features in the Su-Schrieffer-Heeger model by highlighting the "pair-wise" (i.e. chiral) symmetry:

- A topological transition in the bulk can be detected by a change in the pattern of maxima and minima bond orders with first-neighbours, which reflects the two resonance forms

- The chiral symmetry is present if bond orders with second-neighbours are zero in the bulk (i.e. equivalent to the absence of delocalization in meta carbons in benzene

- Topologically protected edge states can be detected by a non-zero second-neighbour bond order at both edges of the molecule. These bond orders decay exponentially to zero far from the edges.

Although the model is simple, we have shown that the approach presented in this work enables chemists to understand the properties of real systems such as black phosphorous or graphene ribbons, and even to construct hand-waving arguments that would extend this model to higher dimensions and more resilent (e.g. time reversal) symmetries.

\section{Acknowledgement}

We really want to thank Francesca Peccati for her patience and help with the images.

The authors acknowledge financial support from ECOS-CONICYT \#C17E09, FONDECYT \#1150806, CONICYT \#21151207 and Programa Basal para Centros de Excelencia de Conicyt, Proyecto FB0807, CEDENNA. AMP thanks the Spanish MINECO, grant CTQ2015-65790-P, the FICyT, grant GRUPIN14-049, and the European Union FEDER funds. JCG thanks 
CALSIMLAB under the public grant ANR-11-LABX0037-01 overseen by the French National Research Agency (ANR) as part of the Investissements d'Avenir program (reference: ANR-11-IDEX-0004-02).

\section{References}

[1] Y. Ando. Topological insulator materials. J. Phys. Soc. Jpn., 82(10):102001, 2013.

[2] J. K. Asbóth, L. Oroszlány, and A. Pályi. A short course on topological insulators. Springer, 2016.

[3] R. F. Bader. Atoms in molecules. Wiley Online Library, 1990.

[4] B. A. Bernevig, T. L. Hughes, and S.-C. Zhang. Quantum spin hall effect and topological phase transition in hgte quantum wells. Science, 314(5806):1757-1761, 2006.

[5] B. A. Bernevig and S.-C. Zhang. Quantum spin hall effect. Phys. Rev. Lett., 96:106802, 2006.

[6] B. Bradlyn, L. Elcoro, J. Cano, M. Vergniory, Z. Wang, C. Felser, M. Aroyo, and B. A. Bernevig. Topological quantum chemistry. Nature, 547:298305, 2017.

[7] R. J. Cava, H. Ji, M. K. Fuccillo, Q. D. Gibson, and Y. S. Hor. Crystal structure and chemistry of topological insulators. J. Mater. Chem. C, 1:31763189, 2013.

[8] H. Chen, W. Zhu, D. Xiao, and Z. Zhang. Co oxidation facilitated by robust surface states on aucovered topological insulators. Phys. Rev. Lett., 107(5):056804, 2011.

[9] Y. L. Chen, J. G. Analytis, J.-H. Chu, Z. K. Liu, S.-K. Mo, X. L. Qi, H. J. Zhang, D. H. Lu, X. Dai, Z. Fang, S. C. Zhang, I. R. Fisher, Z. Hussain, and Z.-X. Shen. Experimental realization of a threedimensional topological insulator, bi2te3. Science, 325(5937):178-181, 2009.

[10] P. Delplace, D. Ullmo, and G. Montambaux. Zak phase and the existence of edge states in graphene. Phys. Rev. B, 84:195452, 2011.

[11] L. Fu and C. L. Kane. Topological insulators with inversion symmetry. Phys. Rev. B, 76:045302, 2007.

[12] L. Fu and C. L. Kane. Superconducting proximity effect and majorana fermions at the surface of a topological insulator. Phys. Rev. Lett., 100:096407, 2008.

[13] A. Gallo-Bueno, M. Kohout, and A. Martín Pendás. Decay rate of correlated real-space delocalization measures: Insights into chemical bonding and mott transitions from hydrogen chains. J. Chem. Theory Comput., 12:3053, 2016.
[14] S. Gupta and A. Saxena. A topological twist on materials science. MRS Bulletin, 39(3):265279, 2014.

[15] M. Z. Hasan and C. L. Kane. Colloquium: Topological insulators. Rev. Mod. Phys., 82:3045-3067, 2010.

[16] A. J. Heeger. Nobel lecture: Semiconducting and metallic polymers: The fourth generation of polymeric materials. Rev. Mod. Phys., 73:681-700, 2001.

[17] T. Jungwirth, J. Sinova, A. Manchon, X. Marti, J. Wunderlich, and C. Felser. The multiple directions of antiferromagnetic spintronics. Nat. Phys., page 1, 2018.

[18] C. L. Kane and E. J. Mele. $Z_{2}$ topological order and the quantum spin hall effect. Phys. Rev. Lett., 95:146802, 2005.

[19] A. B. Khanikaev, S. H. Mousavi, W.-K. Tse, M. Kargarian, A. H. MacDonald, and G. Shvets. Photonic topological insulators. Nat. Mater., 12(3):233-239, 2013.

[20] D. Kong and Y. Cui. Opportunities in chemistry and materials science for topological insulators and their nanostructures. Nat. Chem., 3(11):845-849, 2011.

[21] Y. R. Lin-Liu and K. Maki. Two-soliton interaction energy and the soliton lattice in polyacetylene. Phys. Rev. B, 22:5754-5758, 1980.

[22] E. Locane and P. W. Brouwer. Current-induced switching of magnetic molecules on topological insulator surfaces. Phys. Rev. B, 95:125437, 2017.

[23] A. Martín Pendás, J. M. Guevara-Vela, D. Menéndez Crespo, , A. Costales, and E. Francisco. An unexpected bridge between chemical bonding indicators and electrical conductivity through the localization tensor. Phys. Chem. Chem. Phys., 19:1790, 2017.

[24] L. Mchler, H. Zhang, S. Chadov, B. Yan, F. Casper, J. Kübler, S.-C. Zhang, and C. Felser. Topological insulators from a chemists perspective. Angew. Chem. Int. Ed., 51(29):7221-7225, 2012.

[25] C. Niu, Y. Dai, M. Guo, Y. Ma, B. Huang, and M.-H. Whangbo. Tunable topological surface and realization of insulating massive dira c fermion state in bi2te2se with co-substitution. J. Mater. Chem. C, 1:114-120, 2013.

[26] X.-L. Qi and S.-C. Zhang. Topological insulators and superconductors. Rev. Mod. Phys., 83:10571110, 2011.

[27] C. R. Rajamathi, U. Gupta, N. Kumar, H. Yang, Y. Sun, V. Süß, C. Shekhar, M. Schmidt, H. Blumtritt, P. Werner, B. Yan, S. Parkin, 
C. Felser, and C. N. R. Rao. Weyl semimetals as hydrogen evolution catalysts. Adv. Mater., 29(19):1606202, 2017.

[28] C. R. Rajamathi, U. Gupta, K. Pal, N. Kumar, H. Yang, Y. Sun, C. Shekhar, B. Yan, S. Parkin, U. V. Waghmare, et al. Photochemical water splitting by bismuth chalcogenide topological insulators. ChemPhysChem, 18:2322-2327, 2017.

[29] W. P. Su, J. R. Schrieffer, and A. J. Heeger. Solitons in polyacetylene. Phys. Rev. Lett., 42:1698-1701, 1979.

[30] A. Tanaka and T. Sasaki. Focus on materials science of topological insulators and superconductors. Sci. Technol. Adv. Mater., 16(1):010301, 2015.

[31] G. van Miert, C. Ortix, and C. M. Smith. Topological origin of edge states in two-dimensional inversion-symmetric insulators and semimetals. $2 D$ Materials, 4(1):015023, 2017.

[32] X. Wang, G. Bian, T. Miller, and T.-C. Chiang. Fragility of surface states and robustness of topological order in $\mathrm{bi}_{2} \mathrm{Se}_{3}$ against oxidation. Phys. Rev. Lett., 108:096404, 2012.

[33] Z. Wang, Z. Liu, and F. Liu. Organic topological insulators in organometallic lattices. Nat. Comunn., 4:1471, 2013.

[34] Y. Xia, D. Qian, D. Hsieh, L. Wray, A. Pal, H. Lin, A. Bansil, D. Grauer, Y. Hor, R. Cava, and M. Hasan. Observation of a large-gap topologicalinsulator class with a single dirac cone on the surface. Nat. Phys., 5(6):398-402, 2009.

[35] J. Xiao, L. Kou, C.-Y. Yam, T. Frauenheim, and B. Yan. Toward rational design of catalysts supported on a topological insulator substrate. Acs Catal., 5(12):7063-7067, 2015.

[36] Q.-K. Xue. Nanoelectronics: A topological twist for transistors. Nat. Nanotech., 6(4):197-198, 2011.

[37] J. Yuen-Zhou, S. K. Saikin, N. Y. Yao, and A. Aspuru-Guzik. Topologically protected excitons in porphyrin thin films. Nat. Mater., 13(11):10261032, 2014.

[38] H. Zhang, C.-X. Liu, X.-L. Qi, X. Dai, Z. Fang, and S.-C. Zhang. Topological insulators in bi2se3, bi2te3 and sb2te3 with a single dirac cone on the surface. Nat. Phys., 5(6):438-442, 2009.

[39] W. Zhang, W. Gao, X. Zhang, Z. Li, and G. Lu. Surface spintronics enhanced photo-catalytic hydrogen evolution: Mechanisms, strategies, challenges and future. Appl. Surf. Sci., 434:643 - 668, 2018.

[40] Y. Zhang, K. He, C.-Z. Chang, C.-L. Song, L.L. Wang, X. Chen, J.-F. Jia, Z. Fang, X. Dai,
W.-Y. Shan, et al. Crossover of the threedimensional topological insulator bi 2 se 3 to the two-dimensional limit. Nat. Phys., 6(8):584, 2010. 